\title{
Affect Instability in Adults With a Borderline Personality Disorder
}

\author{
Karen Farchaus Stein
}

\begin{abstract}
This study describes the pattern of affect instability in adults with borderline personality disorder (BPD). Clinical histories and the Diagnostic Inventory for Borderlines were used to identify 3 groups: 1$)$ BPD $(N=15)$, 2) Asymptomatic $(N=10)$, and, 3) Non-BPD, Anorexia Nervosa Clinical Control $(N=4)$. An experience sampling procedure (Hormuth, 1986) was used to obtain 50 measures of affect over 10 days. The findings showed that BPD subjects experienced higher levels of unpleasant affects and greater short-term fluctuations in unpleasant affects than the asymptomatic subjects. However, BPD and asymptomatic subjects experienced more fluctuations in the pleasant affects than the AN subjects. These findings support the hypothesis that BPD is associated with a unique pattern of affect dysregulation.

Copyright 1996 by W.B. Saunders Company
\end{abstract}

$\mathbf{A}$ FFECT INSTABILITY is widely recognized as a core feature of borderline personality disorder (BPD). Rapid and extreme shifts in mood from baseline into dysphoria, irritability or anxiety are identified in the Diagnostic and Statistical Manual of Mental Disorders (American Psychiatric Associations, 1994; 1987) as one of the essential criteria for BPD diagnosis. Clinical and theoretical accounts of the disorder suggest that adults with BPD have a limited ability to buffer the impact of stressors, and, consequently, react with marked shifts in affect each time a stressor is encountered (see Ellison \& Adler, 1990; Gunderson \& Phillips, 1991; Liebowitz \& Klein, 1981; Linehan, 1987a).

Despite the broad consensus acknowledging the centrality of affective instability in BPD, remarkably little is known about the pattern of affect fluctuations that characterize this population. To date, most descriptions of affect instability in BPD are based on clinical accounts that broadly describe

From the University of Michigan School of Nursing, Ann Arbor, MI.

This study was funded by NCNR Grant 32NR06307.

Address reprint requests to Karen Farchaus Stein, PhD, RN, University of Michigan, School of Nursing, 400 N. Ingalls, Room 2344, Ann Arbor, MI 48109. E-mail: kfarchau@umich.edu.

Copyright $\odot 1996$ by W.B. Saunders Company 0883-9417/96/1001-0006\$3.00/0 angry outbursts and precipitous episodes of depression (Cowdry, Gardner, O'Leary, Leibenluft, \& Rubinow, 1991). The few research studies that have examined affect instability have used global measures of affect that provide little information about the pattern of specific affect changes. For example, Cowdry et al. measured affect two times daily over a two-week period in four groups of subjects including adults with BPD, adults with major depression, adults with premenstrual syndrome, and normal adult controls (1991). In this study, two measures of affect were used including a visual analog and a 24-point scale both anchored by "best I've ever felt" and "worse I've ever felt." The findings showed that subjects in the BPD group experienced more variability in affect over the two-week period and less consistency in affect state from day-to-day than the other clinical and nonclinical groups. Although the findings provide general support for the notion that adults with BPD experience more short-term variability in affect than other clinical and nonclinical samples, they provide little specific information about the pattern of affect fluctuations.

The lack of detailed knowledge about the specific pattern of affect instability has had important consequences in the diagnosis and treatment of BPD. In a review paper on the utility of the 
Diagnostic and Statistical Manual 3rd-revised (DSM-III-R) criteria for establishing a BPD diagnosis, Gunderson, Zanarini, and Kisiel acknowledged the high prevalence of affect instability in samples of adults with BPD (1991). However, they questioned the extent to which the symptom contributes to the high rate of overlap between BPD and other personality and depressive diagnoses. They argued that the symptom, as it is currently defined, lacks specificity and suggested that the definition must be refined to improve the usefulness of the symptom in establishing a differential diagnosis.

The lack of clarity in defining the nature of affect instability in BPD has also impacted the development of effective affect-stabilizing interventions. For example, studies on the pharmacotherapy of BPD define affect instability in a variety of ways ranging from rejection sensitivity and irritability to depressive/hypomanic mood swings, and have identified a broad array of psychotropic agents including lithium, monoamine oxidase inhibitors (MAOI), and haloperidol to reduce or eliminate the symptom (for examples, see Cowdry \& Gardner, 1988; Ellison \& Adler, 1990; Links, Steiner, Boiago, \& Irwin, 1990; Soloff, George, Nathan, Schulz, \& Cornelius, 1988). Cognitive-behavioral approaches have tended to view affect instability in BPD as pathologically high levels of emotional reactivity to events and have proposed a variety of foci for intervention including helping individuals reduce the intensity of their reactions to events (see Beck \& Freeman, 1990) and strengthening their capacity to tolerate and cope with intense negative affects (Linehan, 1987a; MacLeod, Williams, \& Linehan, 1992).

Clearly, affect instability is recognized as an important focus of treatment in most approaches to the treatment of $\mathrm{BPD}$, yet the specific affects experienced and the pattern of affect dysregulation remains unclear. Do persons with BPD experience variability in only negative affects, such as sadness or anger and anxiety? Or do they have difficulty regulating all affect states including positive and negative affects? Do adults with BPD differ from other populations according to the frequency of their emotional reactions, the intensity of emotion experienced, or in their ability to restore a baseline level of affect after a threat is encountered? Identification of specific properties of affect dysregulation that are unique to BPD not only holds the potential of improving the diagnostic utility of the symptom but may enable clinicians to develop more focused biologic and cognitive-behavioral affect stabilizing interventions.

The purpose of this study is to describe the pattern of affect instability in adults with BPD and to compare the pattern with those experienced by other asymptomatic and non-BPD clinical samples.

\section{METHODS}

\section{Sample}

Three groups of subjects participated in this study, the Borderline Personality Disorder (BPD) Group $(N=15)$, the Anorexia Nervosa (AN) group $(N=4)$, and the Asymptomatic Control Group $(N=10)$. The AN group was included as a clinical control group to enable investigation of patterns of affect that distinguish adults with BPD from those with other non-BPD psychiatric disorders. Anorexia nervosa was selected for the comparison group because: (A) instability of affect is recognized as an important symptom of both disorders (Cowdry et al., 1991; Goodsitt, 1983) and (B) the severity of the disorders are comparable in that both are associated with severe impairments in self-care abilities that often necessitate inpatient hospitalization.

Subjects for the clinical groups were recruited from three inpatient psychiatric units at local hospitals. The Diagnostic Inventory For Borderlines (DIB) (Gunderson, Kolb, \& Austin, 1981) and a clinical history were used to establish primary diagnosis and group placement. To establish a diagnosis of BPD, the individual had to meet DSM-III-R (American Psychological Association, 1987) criteria for BPD and score 7 or above on the DIB. Similarly, to establish a diagnosis of AN, the individual had to meet $D S M-I I I-R$ criteria for AN and score 5 or below on the DIB. Individuals were considered asymptomatic if they had a negative history for psychiatric disorders and scored 5 or below on the DIB. Additional criteria for participation in the two groups included: (A) no history of organic, developmental, and chronic psychotic disorders and (B) literacy in English.

The DIB is a semi-structured interview that focuses on five areas of functioning including social adaptation, impulse/action patterns, psychotic symptomology, and interpersonal relations (Gunderson \& Kolb, 1978). Based on the subjects' responses to 123 questions, the interviewer rates the subject on 29 items that are summed to 
determine the score. DIB scores range from 0 to 10 with a score of 7 or above necessary for the diagnosis of BPD. Discriminate and known group techniques have been used to support the validity of the measure (Soloff \& Ulrich, 1981).

The DIB was designed to be administered by a skilled psychiatric clinician (Cornell, Silk, Ludolph, et al., 1983). In this study, approximately half of the subjects in the BPD $(N=8)$ were recruited from an ongoing study of persons with BPD (Silk, Lee, Hill, \& Lohr, 1995). For these subjects, the DIB was administered by a member of the personality disorders research team before entry into this study. An acceptable level of interrater reliability for the team was established $(\kappa=.80)$ (Baker, Silk, Weston, Nigg, \& Lohr, 1992). For all other subjects, the DIB was administered by the investigator, who was trained in the administration of the DIB. The investigator was considered a reliable interviewer after concurrently rating five patients with a member of the personality disorders research team and successfully establishing: 1) the same group placement and 2) a total DIB score that was within one scale point as that assigned by the experienced interviewer.

Twelve of the 15 subjects in the BPD group, nine of the 10 Asymptomatic subjects, and all four of the AN group were female. The mean age was 27.6 $(S D=5.7)$ years for the BPD group, 29.3 $(S D=7.0)$ years for the Asymptomatic group and $26.3(S D=11.3)$ years for the AN group $(p=n s)$. Subjects in the Asymptomatic group had higher level of education $(M=17.2, S D=2.5)$ than those in the BPD group $(M=14.2, S D=3.6)$ and the AN group $(M=13.8, S D=1.5$; ANOVA with Tukey's Honestly Significant Different Test (HSD), $p<.05$ ). Tukey's HSD, was used for all post hoc comparisons because this statistic adjusts the alpha level to reflect the number comparisons performed. In the BPD group, 8 subjects were single, 1 married, and 6 divorced. In the Asymptomatic group, 4 subjects were single and 6 were married. In the AN group, 3 subjects were single and 1 subject was married.

Six of the BPD subjects were not on psychotropic medications at the time of data collection. Of the 9 on psychotropic medications, 4 were on a mood-stabilizing drug, 2 were on antidepressants, 1 was on an antipsychotic medication, and 2 were receiving a combination of antidepressant, moodstabilizing and antipsychotic drugs. Of the 9 sub- jects on medication, 6 subjects had started the medication during the current hospitalization and 3 subjects had begun the medication before admission. In the AN Group, 2 subjects were not on psychotropic medication and 2 had started taking medication during the current hospitalization. One subject was taking both a mood-stabilizing and an antidepressant medication and the other was taking an antipsychotic medication.

\section{Measures}

To examine instability or short-term fluctuations in affect, it was necessary to measure affect states repeatedly over time. The experience sampling method (Hormuth, 1986; Larson \& Csikszentmihalyi, 1983) was used to obtain multiple measures of affect during the person's everyday activities. Subjects were asked to wear an alarm watch for a period of 10 days. The watch was set to signal the subject five times daily at random intervals approximately 3 hours in length between the hours of 9 a.m. and 9 p.m. During the experience sampling period, subjects carried a small notebook of diaries with them. At each signal, subjects were instructed to immediately complete a one-page, diary-type questionnaire that included a measure of affect.

Instability of Affect. The Self-Report Affect Circumplex Scale (Larsen \& Diener, 1992) was used to measure instability of affect. This measure is based on the circumplex model of affect in which it is posited that two main dimensions are underlying the majority of affect states: hedonic valence (pleasant and unpleasant dimension) and activation (high- and low-energy dimension). Four additional dimensions of affect are identified that reflect combinations of the two main dimensions. They include: (A) activated-pleasant, (B) unactivatedpleasant, (C) activated-unpleasant, and (D) unactivated-unpleasant affects. Subjects are asked to rate 48 mood adjectives on a 5-point scale according to "how you are feeling now." The scale was anchored by "not at all/slightly" at one pole and "extremely" at the other. Eight affect scales, each consisting of 6 adjectives, are included in the measure (see Figure 1). Cronbach alpha coefficientsfor the scales ranged from .70 to .94 .

For this study, the within-subject standard deviation across the repeated measures of the affect state was used as an index of instability of the affect. For each of the 8 affect scales, the subject's standard 


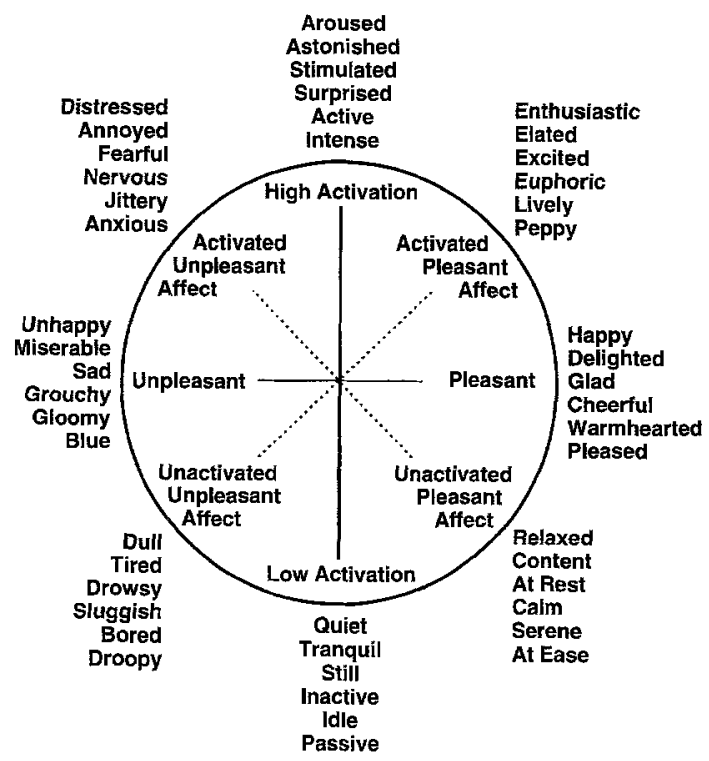

Fig 1. The self-report affect circumplex. Larsen, R., \& Diener, E. Review of personality and social psychology promises and problems with the circumplex model of emotion (13), pp 25-29, copyright 1992 by Sage Publications, Inc. Reprinted by permission of Sage Publications, Inc.

deviation across the multiple measures of the affect was computed. The individual's standard deviation on the affect scale was then used to calculate the group mean standard deviation.

Level of Affect. Responses on the Self-Report Affect Scale (Larsen \& Diener, 1992) were also used to measure the average level of each of the 8 affect states. For each of the 8 scales, the subject's mean score across the multiple measures of the affect was computed and used as an index of level of the affect state. Again, the individual's mean scores on each of the 8 affect scales were used to compute group mean level scores.

\section{Procedures}

Approval for the study was obtained from the Institutional Review Board at the University of Michigan. All subjects completed a written informed consent before participating in the study. For all subjects, except the BPD subjects who were recruited from the personality disorder project, the DIB was administered by the investigator. To ensure that acute cognitive changes associated with starvation did not confound measurement of the study variables, individuals with anorexia nervosa were recruited into the study after they had successfully completed nutrition classes and a nutrition examination. These two components of the treatment protocol indicate an ability to process and retain complex information and, therefore, were used as markers of an acceptable level of cognitive functioning. Subjects for the Asymptomatic control group were recruited through written advertisements from the community.

Approximately three days after completing the DIB interview, subjects completed a packet of written questionnaires not addressed in this report. Subjects were then oriented to the experience sampling procedure and instructed on the completion of the diary. A member of the research team met with the subject approximately every three days during the 10-day experience sampling period to reset alarm times, respond to any questions, and to collect completed diaries. The alarm times were changed two times during the study to reduce the possibility that subjects would become accustomed to the signal times and alter their routines in anticipation. Completed diaries were collected to reduce the chance of a subject completing all diaries at once near the end of the study. Finally, it was hoped that frequent meetings with a member of the research team would increase the diary response rate.

Each subject was signaled a total of fifty times over the 10-day period. All subjects in the BPD and AN groups began the experience sampling during inpatient hospitalization. For those subjects who were discharged before the end of the experience sampling, data collection continued during their return to community living. For the BPD group, $55 \%$ of the diaries were completed during inpatient hospitalization. For the AN group, 69\% were completed during inpatient hospitalization. Response rate for diaries was $73 \%$ for the BPD group, $83 \%$ for the AN group, and $88 \%$ for the Asymptomatic group. Three subjects, all in the BPD group, failed to complete at least 15 repeated measures of affect. Consequently, their data was dropped from all analyses. For the remaining subject, the mean number of logs completed in the BPD group was $42.7(S D=11.7$; range $=15-50)$, AN group was $49.0(S D=1.7$; range $=32-50)$, and the Asymptomatic group was $43.9(S D=8.2$, range $=27$ 50 ). Subjects were paid $\$ 5$ for completing the DIB and an additional $\$ 35$ for completing the rest of the study protocol. 


\section{RESULTS}

Instability of Affect

A univariate analysis of variance model (ANOVA) with Tukey's Honestly Significant Difference test (HSD) were used to examine group differences in instability of the eight affect states. Table 1 shows the mean instability scores for the three study groups and results of the ANOVAs.

Results of the ANOVAs showed significant group differences in 5 of the 8 affect instability scores including unactivated-pleasant (excited/lively), high activation (surprised/aroused), unpleasant (sad/ unhappy), activated-unpleasant (anxious/fearful), and unactivated-unpleasant (bored/tired) affects. Post-hoc comparisons showed an interesting pattern of findings. Subjects in the BPD group had higher instability scores than the Asymptomatic group in 3 affects including unpleasant (sad/ unhappy), activated-unpleasant (anxious/fearful), and unactivated-unpleasant (bored/tired) affects. When compared to the AN group, the BPD group had a higher instability score for high-activation affect (surprised/aroused), but the two groups did not differ in the level of instability for the 3 unpleasant affect scales.

A very different picture emerged, however, for the pleasant and low activation affect scales. For these affects, BPD and Asymptomatic subjects looked remarkably similar with both groups reporting approximately equal levels of fluctuation in pleasant (happy/cheerful), activated-pleasant (excited/lively), unactivated-pleasant (relaxed/content), and low activation (quiet/tranquil) affects. In contrast, AN subjects reported significantly less instability in unactivated-pleasant (relaxed/content) affect than both the Asymptomatic and BPD groups. In addition, AN subjects showed a nonsig-

Table 1. Means and Results of ANOVAs of 8 Affect Instability Scores for BPD, Asymptomatic, and AN Groups

\begin{tabular}{lllllll}
\hline \multicolumn{1}{c}{ Affect } & BPD & Asymp & AN & & \\
& $N=12$ & $N=10$ & $N=4$ & \multicolumn{1}{c}{$F$} & \multicolumn{1}{c}{$p$} \\
\hline Pleasant & .77 & .79 & .37 & 2.80 & .082 \\
Activated pleasant & .70 & .62 & .31 & 3.11 & .064 \\
Unactivated pleasant & $.73^{\mathrm{a}}$ & $.74^{\mathrm{b}}$ & $.25^{\mathrm{ab}}$ & 4.64 & .020 \\
Low activation & .81 & .74 & .47 & 2.87 & .077 \\
High activation & $.65^{\mathrm{a}}$ & .43 & $.31^{\mathrm{a}}$ & 5.08 & .015 \\
Unpleasant & $.85^{\mathrm{a}}$ & $.27^{\mathrm{ab}}$ & $.77^{\mathrm{b}}$ & 34.80 & $<.001$ \\
Activated unpleasant & $.83^{\mathrm{a}}$ & $.33^{\mathrm{a}}$ & .65 & 12.58 & $<.001$ \\
Unactivated unpleasant & $.78^{\mathrm{a}}$ & $.54^{\mathrm{a}}$ & .59 & 3.36 & .052 \\
\hline
\end{tabular}

Note. Groups that share the same superscripts are significantly different at $p \leq .05$ using Tukey-HSD.

\begin{tabular}{|c|c|c|c|c|c|}
\hline Affect & $\begin{array}{c}\text { BPD } \\
N=12\end{array}$ & $\begin{array}{l}\text { Asymp } \\
N=10\end{array}$ & $\begin{array}{c}\text { AN } \\
N=4\end{array}$ & $F$ & $p$ \\
\hline Pleasant & .09 & .07 & .02 & 1.09 & .350 \\
\hline Activated pleasant & .06 & .04 & .00 & 1.03 & .372 \\
\hline Unactivated pleasant & .06 & .06 & .00 & 1.32 & .287 \\
\hline Low activation & .08 & .09 & .00 & 1.95 & .165 \\
\hline High activation & .03 & .00 & .00 & 3.31 & .055 \\
\hline Unpleasant & $.06^{\mathrm{a}}$ & $.00^{\mathrm{a}}$ & .04 & 5.37 & .012 \\
\hline Activated unpleasant & .06 & .01 & .04 & 2.25 & .129 \\
\hline Unactivated unpleasant & .08 & .03 & .02 & 2.31 & .122 \\
\hline
\end{tabular}

Note. Groups that share the same superscripts are significantly different at $p \leq .05$ using Tukey-HSD.

nificant tendency toward lower instability scores on the pleasant (happy/cheerful) and activated-pleasant (excited/lively) scales.

To further explore the nature of affect instability in adults with BPD, a more refined exploration of the pattern of affect shifts was examined. Larsen (1987) has suggested that the within-subject standard deviation is an aggregate statistic, which when used as an index of instability of affect, does not separate frequency of affect change from extremity of those changes. For example, it is impossible to distinguish extreme and frequent affect shifts from extreme but slow shifts in affect, based on the within-subject standard deviation alone.

To further explore the pattern of affect shifts that characterize this population a score was computed to reflect the proportion of consecutive affect ratings with a change of 2 points or more. To compute this score, the affect scale score at $T_{x}$ was subtracted from the affect score at $T_{x+1}$. When the absolute value of the difference score was greater than or equal to 2, a value of 1 was assigned; otherwise a value of 0 was assigned. The sum of the difference scores were then divided by the total number of difference scores computed for the individual on that affect scale. The individual's proportion scores were then used to compute group mean proportions and ANOVAs were used to examine group differences. The mean proportions by group and results of the ANOVAs are shown in Table 2. As can be seen, BPD subjects experienced a significantly greater proportion of 2 point changes in unpleasant (sad/unhappy) affect than those in the Asymptomatic group. In addition, the BPD subjects displayed a nonsignificant tendency toward a higher proportion of 2 point changes in the activatedunpleasant $(M=.06, S D=.07$, Range $=0-.20)$ 
and unactivated-unpleasant domains $(M=.08$, $S D=.09$, Range $=0-.28$ ) when compared to the Asymptomatic controls (activated-unpleasant: $M=.01, S D=.02$, Range $=0-.06$; unactivatedunpleasant: $M=.03, S D=.03$, Range $=0-.10$ ).

\section{Level of Affect}

Univariate analysis of variance with Tukey's HSD post-hoc comparisons were also used to examine group differences in mean levels of the 8 affect states. Mean level affect scores for the 3 study groups along with results of the ANOVAs are shown in Table 3 .

Consistent with current clinical conceptions of BPD, subjects in the BPD group reported significantly higher mean levels of unpleasant (sad/ unhappy) and activated-unpleasant (anxious/fearful) affect than subjects in the Asymptomatic group.

No significant differences were found between the BPD and AN groups on any of the 8 affect levels scores. However, AN subjects were distinguished from the Asymptomatic subjects in that they reported significantly lower levels of pleasant (happy/cheerful) and unactivated-pleasant (relaxed/ content) affects and higher levels of unpleasant (sad/unhappy) and activated-unpleasant (anxious/ fearful) affects.

\section{Persistence of Affect}

Another important question related to the affective experience of adults with BPD is whether they have difficulty restoring affect states to a baseline level after an increase in level occurs. To examine group differences in the restoration of affect to baseline levels, first order autocorrelation coefficients for each of the eight affect scales were examined. A first order autocorrelation coefficient

\begin{tabular}{|c|c|c|c|c|c|}
\hline Affect & $\begin{array}{c}\text { BPD } \\
N=12\end{array}$ & $\begin{array}{l}\text { Asymp } \\
N=10\end{array}$ & $\begin{array}{c}\text { AN } \\
N=4\end{array}$ & $F$ & $p$ \\
\hline Pleasant & 2.06 & $2.52^{\mathrm{a}}$ & $1.51^{\mathrm{a}}$ & 3.86 & .036 \\
\hline Activated Pleasant & 1.75 & 1.71 & 1.27 & 1.55 & .233 \\
\hline Unactivated Pleasant & 2.02 & $2.59^{a}$ & $1.58^{\mathrm{a}}$ & 5.35 & .012 \\
\hline Low Activation & 2.09 & 1.99 & 1.88 & 0.63 & .542 \\
\hline High Activation & 1.89 & 1.62 & 1.24 & 3.28 & .056 \\
\hline Unpleasant & $2.12^{\mathrm{a}}$ & $1.12^{\mathrm{ab}}$ & $2.64^{b}$ & 10.03 & $<.001$ \\
\hline Activated Unpleasant & $2.29^{a}$ & $1.23^{\mathrm{ab}}$ & $2.85^{b}$ & 10.03 & $<.001$ \\
\hline Unactivated Unpleasant & 1.92 & 1.55 & 2.12 & 2.35 & .118 \\
\hline
\end{tabular}

Note. Groups that share the same superscripts are significantly different at $p \leq .05$ using Tukey-HSD.

$\begin{gathered}\text { Table 4, Mean Scores and ANONA's on Fischer's Z } \\
\text { Transformed Autocorrelation Coefficient Scores }\end{gathered}$
\begin{tabular}{lcccccc}
\multicolumn{1}{c}{ Affect } & BPD & Asymp & AN & & \\
\multicolumn{1}{c}{$N=12$} & $N=10$ & $N=4$ & $F$ & $\rho$ \\
\hline Pleasant & 0.33 & 0.19 & 0.24 & 0.79 & .467 \\
Activated Pleasant & 0.29 & 0.19 & 0.41 & 1.15 & .333 \\
Unactivated Pleasant & 0.27 & 0.19 & 0.23 & 0.45 & .642 \\
Low Activation & 0.21 & 0.11 & 0.30 & 3.29 & .056 \\
High Activation & 0.28 & 0.17 & 0.23 & 0.80 & .460 \\
Unpleasant & 0.45 & 0.30 & 0.43 & 0.93 & .411 \\
Activated Unpleasant & 0.34 & 0.22 & 0.27 & 1.14 & .336 \\
Unactivated Unpleasant & $0.20^{\mathrm{a}}$ & $0.16^{\mathrm{b}}$ & $0.45^{\mathrm{ab}}$ & 4.35 & .025
\end{tabular}

Note. Groups that share the same superscripts are significantiy different at $p \leq .05$ using Tukey-HSD.

provides an index of how well scores at time $T_{x}$ correlate with scores at $T_{x+1}$. As such, the autocorrelation coefficient provides an indicator of the magnitude of the persistence of an affect state between two measurement points (Huba, Lawlor, Stallone, \& Fieve, 1976).

To investigate group differences in persistence of affect, the individual's first order autocorrelation coefficient was calculated for each of the 8 affect scales. To meet the assumption of a normal distribution underlying the ANOVA model (Fox, 1984) the individual's autocorrelation coefficients were transformed to Z-scores using Fisher's Transformation procedure (Volicer, 1984). Group mean autocorrelation coefficients were then computed and group differences were examined using the one-way ANOVA statistic. Tukey-HSD test was used for post-hoc comparisons. Table 4 shows the mean Fischer's Z-transformed autocorrelation coefficients by group and results of the ANOVAs.

Subjects in the BPD group did not differ significantly from the Asymptomatic subjects nor AN subjects in the persistence of any of the 8 affect states. However, AN subjects experienced significantly more persistence of unactivated-unpleasant (bored/tired) affect than the Asymptomatic subjects.

\section{DISCUSSION}

The findings of this study offer preliminary support for the idea that borderline personality disorder is associated with a unique pattern of affect dysregulation. Consistent with the DSMIII- $R$ description that characterizes affect instability in BPD as "marked shifts from baseline mood to depression, irritability, or anxiety" (APA, 1987), BPD subjects in this study reported both higher levels of unpleasant (sad/unhappy) and activated- 
unpleasant (anxious/fearful) affects and greater fluctuations in the unpleasant affects states than Asymptomatic adults. Furthermore, the higher proportion of consecutive ratings of unpleasant affect with a change of at least two points found within this group provide some evidence to suggest that the fluctuations were short-term in nature occurring over intervals of a few hours rather than days.

It is particularly interesting to note that although the BPD group's instability scores for the pleasant (happy/cheerful), activated-pleasant (excited/lively), and unactivated-pleasant (relaxed/content) states were approximately equal to those found for the 3 unpleasant affects, they were not meaningfully different from the pleasant affects instability scores of the asymptomatic controls. Asymptomatic subjects experienced considerable fluctuations in pleasant (happy/cheerful), activated-pleasant (excited/ lively), and unactivated-pleasant (relaxed/content) scale scores with the average variability around the mean, approximately $75 \%$ of a full scale point. This level of variability in the pleasant affect is in marked contrast to the smaller standard deviations observed for the unpleasant affects in this group. When taken together, these findings suggest that peaks and dips in happiness, excitement, or contentment that last for a period of a few hours within one's day are a part of the normal affective experience of adults in our culture. Furthermore, the lack of significant differences between the Asymptomatic and BPD groups provides evidence to suggest that the regulation of pleasant affects in BPD is unimpaired.

In contrast to the BPD group whose affect dysregulation seemed to be focused in the unpleasant affects alone, subjects in the AN group displayed significant differences in both pleasant and unpleasant affect states. AN subjects were like the BPD group in that they experienced higher levels of unpleasant (sad/unhappy) and activated-unpleasant (anxious/fearful) affects and greater fluctuations in unpleasant affects than the Asymptomatic subjects. However, AN subjects were further distinguished from the Asymptomatic controls in that they also reported lower levels of pleasant (happy/ cheerful) and activated-pleasant (excited/lively) affects and less fluctuation in the unactivatedpleasant (relaxed/content) affect domain. Although the sample size for this group in particular was small and consequently, replication with a larger sample is necessary, these findings offer preliminary evidence to suggest that affect dysregulation may assume different forms across the various psychiatric diagnoses. In contrast to the view that affect instability as a diagnostic indicator may contribute to the overlap among various disorders (Gunderson, Zanarini, \& Kisiel, 1991), these findings offer preliminary evidence to suggest that detailed evaluation of the pattern of regulation across the full range of affects may provide valuable information about subtle differences in affect dysregulation associated with each of the disorders.

Another striking finding of this study is that although significant group differences were found between the BPD and Asymptomatic subjects in the level of unpleasant (sad/unhappy) and activatedunhappy (anxious/fearful), the average level of unpleasant affects reported by the BPD group was remarkably low. Many theoretical and clinical accounts suggest that not only are adults with BPD highly sensitive to emotional stimuli but, in addition, their emotional reactions are pathologically intense and slow to return to baseline (Linehan, 1987b; Snyder \& Pitt, 1985; Soloff \& Ulrich, 1981; Soloff, Cornelius, \& George, 1991). Based on this perspective, individuals with BPD could be expected to report higher average levels of anger, irritability, and sadness. Yet in this sample of adults with BPD, the mean scores on the 3 unpleasant affect scales ranged from 1.92 to 2.29 , corresponding to the points on the scale anchored by "a little" to "moderate" level of intensity. Although the fact that over half of the sample was on at least one psychotropic medication and a substantial proportion of the measurements of affect were made during inpatient hospitalization could account for the relatively low levels of unpleasant affect reported by this group, the findings do raise questions about the level of sad/unhappy and anxious/fearful affects routinely experienced by this population (see footnote on p. 39).

Another unexpected finding of this study was that BPD subjects did not differ from those in the Asymptomatic group in the persistence of unpleasant affects across time. This finding offers a preliminary challenge to the hypothesis that adults with BPD have difficulty restoring affect to baseline levels. Several theorists have speculated that deficits in the early nurturing environment impede the development of self-soothing abilities necessary to regulate and control negative affects once they have been triggered (Kernberg, 1980; Linehan, 1987b). From this perspective, anger, sadness, and anxiety are not only more easily triggered in those with 
BPD, but in addition, these unpleasant affects persist for longer intervals because of the individual's limited ability to self-soothe and restore positive affect states. In contrast to this theoretical perspective, the BPD subjects in this study, like those in the Cowdry study (Cowdry et al., 1991), showed relatively low levels of persistence of the unpleasant affects that were not distinguished from those observed in the asymptomatic controls (see Huba et al., 1976 for example of high persistence of unpleasant affects in depression).

The establishment of a foundation of knowledge about the nature of affect instability is a critical first step toward the development of empirically-based intervention strategies that will strengthen the nurse's ability to promote adaptive functioning in adults with a borderline personality disorder. Currently, intervention strategies to enhance affect regulation in adults with BPD are broadly focused on diverse aspects of the affective experience, including strategies to decrease the emotional response to stimuli (Beck \& Freeman, 1990), to strengthen the individual's ability to cope with intense levels of affect (Kaplan, 1986), and to improve self-soothing capacities (Linehan, 1987b). Although the sample sizes in this study are small and replications with community-based samples are needed, the findings provide preliminary evidence to suggest that an important focus in the treatment of affect dysregulation associated with BPD is the short-term fluctuations in sad/unhappy and anxious/fearful affects. Nursing interventions designed to identify stimuli that trigger these emotional reactions and desensitize the individual to the critical events may play an important role in stabilizing affect and improving feelings of wellbeing. Diaries, such as the one used in this study, could be used to track fluctuations in unpleasant affects and to identify the specific stimuli that precipitated the shift. Cognitive therapy strategies used within the context of supportive and caring therapeutic relationship (Nehls, 1994) could then be used to further clarify the meaning of the stimulus to the individual and to identify alternative ways to frame the event. Working effectively with adults with BPD to stabilize affect and enhance emotional well-being rests firmly on nursing's ability to gain a refined understanding of the nature of the affective experience in BPD and our ability to translate empirical knowledge into effective intervention strategies.

\section{ACKNOWLEDGMENT}

The author acknowledges the contributions of David Ronis, $\mathrm{PhD}$, and Cathy Antonakos, $\mathrm{PhD}$, in the data analysis for this paper.

\section{FOOTNOTE}

To examine the effects of psychotropic medications on level and instability of affect, the BPD subjects were divided into 2 groups [BPD subjects on medications $(N=6)$ and BPD subjects on no medications $(N=4)]$ and the levels and instability scores were compared using one-way ANOVAs. No significant differences were found between the groups in level or instability of the 8 affect states. These results, however, must be viewed as tentative because the sample sizes were small and the medication group was heterogeneous with individuals on a variety of psychotropic drugs for varying lengths of time.

\section{REFERENCES}

American Psychiatric Association (1994). Diagnostic and statistical manual of mental disorders (4th ed.). Washington, DC: Author.

American Psychiatric Association (1987). Diagnostic and statistical manual of mental disorders (3rd ed.). Washington, DC: Author.

Baker, L., Silk, K.K., Weston, D., Nigg, J., \& Lohr, N. (1992). Malevolence, splitting, and parental ratings by borderlines. Joumal of Nervous and Mental Disease, 180, 258-264.

Beck, A.T., \& Freeman, A. (1990). Cognitive therapy of personality disorders. New York: Guilford.

Cornell, D.G., Silk, K.K., Ludolph, P.S., et al. (1983). Test-tetest reliability of the diagnostic interview for borderlines. Archives of General Psychiatry, 40, 1307-1310.

Cowdry, R.W., \& Gardner, D. (1988). Pharmacotherapy of borderline personality disorder. Archives of General Psychiatry, 45, 111-119.

Cowdry, R.W., Gardner, D., O'Leary, K., Leibenluft, E., \& Rubinow, D. (1991). Mood variability: A study of four groups. American Journal of Psychiatry, 148, 15051511.

Ellison, J., \& Adler, D. (1990). A strategy for the pharmacology of personality disorders. New Directions for Mental Health Services, 47, 43-60.

Fox, J. (1984). Linear statistical models and related methods. New York: John Wiley.

Goodsitt, A. (1983). Self-regulatory disorders in eating disorders. International Journal of Eating Disorders, 2 51-61.

Gunderson, J., \& Kolb, J. (1978). Discriminating features of borderline personality. American Journal of Psychiatry, $135,792-796$.

Gunderson, J., Kolb, J., \& Austin, V. (1981). The diagnostic interview for borderlines. American Journal of Psychiatry, 138, 896-903.

Gunderson, J., \& Phillips, K. (1991). A current view of the interface between borderline personality disorder and depression. American Journal of Psychiatry, 148, 967 974. 
Gunderson, J., Zanarini, M., \& Kisiel, C. (1991). Borderline personality disorder: A review of data on DSM-III-R descriptions. Journal of Personality Disorders, 5, 340353.

Hormuth, S.E. (1986). The sampling of experience in situ. Journal of Personality, 54, 261-293.

Huba, G.J., Lawlor, W.G., Stallone, F., \& Fieve, R. (1976). The use of autocorrelation analysis in the longitudinal study of mood patterns in depressed patients. British Journal of Psychiatry, 128, 146-155.

Kaplan, C. (1986). The challenge of working with patients diagnosed as having a borderline personality disorder. Nursing Clinics of North America, 21, 429-438.

Kernberg, O. (1980). The development of intrapsychic structures in the light of borderline personality organization. In S.I. Greenspan, \& G.H. Pollock (Eds.), The course of life: Psychoanalytic contributions toward understanding personality development: Vol. III. Adulthood and the aging process. (pp. 349-365). NIMH.

Larsen, R. (1987). The stability of mood variability: A spectral analytic approach to daily mood assessments. Journal of Personality and Social Psychology, 52, 1195-1204.

Larsen, R., \& Diener, E. (1992). Promises and problems with the circumplex model of emotion. Review of Personality and Social Psychology, 13, 25-59.

Larson, R., \& Csikszentmihalyi, M. (1983). The experience sampling method. In H.T. Reis (Ed.), Naturalistic ap. proaches to studying social interaction. San Francisco: Jossey-Bass.

Linehan, M. (1987a), Dialectical behavioral therapy: A cognitive behavioral approach to parasuicide. Journal of Personality Disorders, 1, 328-333.

Linehan, M. (1987b). Dialectical behavior therapy for border- line personality disorder. Bulletin of the Menninger Clinic, 51, 261-276.

Liebowitz, M., \& Klein, D. (1981). Interrelationship of hysteroid dysphoria and borderline personality disorder. Psychiatric Clinics of North America, 4, 67-87.

Links, P., Steiner, M., Boiago, I., Irwin, D. (1990). Lithium therapy for borderline patients: Preliminary findings. Journal of Personality Disorders, 4, 173-181.

MacLeod, A., Williams, J., Linehan, M. (1992). New developments in the understanding and treatment of suicidal behavior. Behavioral Psychotherapy, 20, 193-218.

Nehls, N. (1994). Brief hospital treatment plans for persons with borderline personality disorder: Perspectives of inpatient psychiatric nurses and community mental health center clinicians. Archives of Psychiatric Nursing, 8, 303-311.

Silk, K.R., Lee, S., Hill, E.M., \& Lohr, N.E. (1995). Borderline symptoms and severity of sexual abuse. American Journal of Psychiatry, 152, 1059-1064.

Soloff, P., Cornelius, J., \& George, A. (1991). Relationship between Axis I and Axis II Disorders: Implications for treatment. Psychopharmacology Bulletin, 27, 23-30.

Soloff, P., George, A., Nathan, S., Schulz, P., Cornelius, J. (1988). Patterns of response to amitriptyline and haloperidol among borderline patients. Psychopharmacology Bulletin, 24, 264-268.

Soloff, P., \& Ulrich, R.F. (1981). Diagnostic interview for borderline patients. Archives of General Psychiatry, 38, 686-692.

Synder, S., \& Pitt, W. (1985). Characterizing anger in the DSM-III borderline disorder. Acta Psychiatry Scandinavia, 52, 464-469.

Volicer, B. (1984). Multivariate statistics for nursing research. New York: Grune \& Stratton. 